\title{
Plasma levels of soluble Axl correlate with severity of community-acquired pneumonia
}

\author{
CHUNG-PO KO ${ }^{1,2^{*}}$, YUNG-LUEN YU ${ }^{3,4^{*}}$, PEI-CHING HSIAO ${ }^{5,6}$, SHUN-FA YANG $^{1,7}$ and CHAO-BIN YEH ${ }^{5,8,9}$ \\ ${ }^{1}$ Institute of Medicine, Chung Shan Medical University; \\ ${ }^{2}$ Department of Neurosurgery, Tungs' Taichung Metro Harbor Hospital; \\ ${ }^{3}$ Graduate Institute of Cancer Biology and Center for Molecular Medicine, China Medical University; \\ ${ }^{4}$ Department of Biotechnology, Asia University; ${ }^{5}$ School of Medicine, Chung Shan Medical University; \\ Departments of ${ }^{6}$ Internal Medicine and ${ }^{7}$ Medical Research, Chung Shan Medical University Hospital; \\ ${ }^{8}$ Department of Emergency Medicine, School of Medicine, Chung Shan Medical University; \\ ${ }^{9}$ Department of Emergency Medicine, Chung Shan Medical University Hospital, Taichung, Taiwan, R.O.C.
}

Received June 13, 2013; Accepted January 30, 2014

DOI: $10.3892 / \mathrm{mmr} .2014 .1933$

\begin{abstract}
The aim of this study was to examine the plasma level changes of soluble Axl (sAxl) prior to and following treatment with antibiotics in hospitalized adult patients with community-acquired pneumonia (CAP), and to investigate the correlating clinical and laboratory manifestations of CAP with plasma sAxl levels. Blood samples were obtained from 61 adult CAP patients (prior to and following treatment with antibiotics) and 60 healthy controls in order to measure the plasma concentrations of sAxl using the enzyme-linked immunosorbent assay. The plasma-soluble Axl concentration level was markedly elevated in patients with CAP prior to treatment, compared with the controls, and decreased markedly following treatment. The levels of white blood cells, neutrophils, and C-reactive protein decreased markedly following treatment with antibiotics and did not correlate with the concentration level of sAxl. However, the plasma concentration of sAxl correlated with the severity of CAP with the pneumonia severity index score $(\mathrm{r}=0.350$, $\mathrm{P}=0.006, \mathrm{n}=61)$, the CURB-65 score $(\mathrm{r}=0.281, \mathrm{P}=0.028, \mathrm{n}=61)$ and the acute physiology and chronic health evaluation II score $(\mathrm{r}=0.313, \mathrm{P}=0.014, \mathrm{n}=61)$. In conclusion, plasma sAxl may be involved in the clinical assessment of the severity of CAP,
\end{abstract}

Correspondence to: Dr Chao-Bin Yeh or Professor Shun-Fa Yang, Department of Emergency Medicine, School of Medicine, Chung Shan Medical University, Section 1, 110 Chien-Kuo N. Road, Taichung 402, Taiwan, R.O.C.

E-mail: sky5ff@gmail.com

E-mail: ysf@csmu.edu.tw

*Contributed equally

Key words: soluble Axl, community-acquired pneumonia, pneumonia severity index score, CURB-65, acute physiology and chronic health evaluation II score which may guide the development of treatment strategies and predict the clinical outcome.

\section{Introduction}

Pneumonia is an inflammatory condition of the lung that involves lung parenchyma. Community-acquired pneumonia (CAP) is one of several diseases that results in lung infections in individuals who have not recently been hospitalized. CAP may be caused by bacterial, viral, fungal or parasitic agents with the additional complication of upper respiratory tract infections $(1,2)$. Furthermore, CAP is the most frequently occurring infectious disease worldwide, with high morbidity and mortality rates (3). Timely diagnosis and optimized antibiotic treatment are critical for reducing CAP morbidity and mortality. Early diagnosis and recognition of the severity of CAP is required for early treatment in order to increase the survival rate of CAP patients (4).

Growth arrest-specific protein 6 (Gas6) is a vitamin K-dependent protein, which was initially described as a protein expressed during growth arrest. Gas6 is also crucial for phagocytosis of apoptotic cells (5-7). Axl is the receptor tyrosine kinase (RTK) for Gas6 and belongs to a subfamily of RTKs (8). Axl is present in the endothelium and is capable of being cleaved and released into circulation; it is crucial for endothelial activation during inflammation. The soluble form of Axl (sAxl), which is present in plasma, binds Gas6 and inhibits Axl-mediated cell signaling. Numerous studies have demonstrated that the Gas6-Axl system is capable of regulating cell survival, proliferation, migration, adhesion and phagocytosis (5-8). Gheita et al (8) indicated that plasma concentrations of sAxl correlate positively with systemic lupus erythematosus (SLE) and Behçet's disease. Ekman et al reported that Gas6 and sAxl correlate with numerous inflammatory markers, suggesting a role in systemic inflammation, including SLE and critical limb ischemia (CLI) diseases $(5,6)$. Therefore, the inflammation severity and survival rate of these diseases may be indirectly predicted by sAxl levels, which 
Table I. Laboratory data of controls and patients with community-acquired pneumonia (CAP) prior to and following treatment . $^{\mathrm{a}}$

\begin{tabular}{|c|c|c|c|c|c|}
\hline \multirow{2}{*}{$\begin{array}{l}\text { Clinical } \\
\text { variables }\end{array}$} & \multirow{2}{*}{$\frac{\text { Controls }(\mathrm{n}=60)}{\text { Median (range) }}$} & \multirow{2}{*}{$\frac{\text { Pre-treatment }(\mathrm{n}=61)}{\text { Median (range) }}$} & \multirow{2}{*}{$\frac{\text { Post-treatment }(\mathrm{n}=61)}{\text { Median (range) }}$} & \multirow{2}{*}{$\begin{array}{l}\text { P-value } \\
\mathrm{UT} / \mathrm{C}^{\mathrm{b}}\end{array}$} & \multirow{2}{*}{$\begin{array}{r}\text { P-value } \\
\mathrm{UT} / \mathrm{T}^{\mathrm{c}}\end{array}$} \\
\hline & & & & & \\
\hline Age (years) & $59.38 \pm 11.45$ & $59.52 \pm 20.46$ & & 0.963 & \\
\hline \multicolumn{6}{|l|}{ Gender } \\
\hline Male & $36(60 \%)$ & $37(60.7 \%)$ & & 0.941 & \\
\hline Female & $24(40 \%)$ & $24(39.3 \%)$ & & & \\
\hline CRP (mg/dl) & $\begin{array}{c}0.30 \\
(0.02-1.65)\end{array}$ & $\begin{array}{c}8.63 \\
(0.69-27.40)\end{array}$ & $\begin{array}{c}0.94 \\
(0.30-11.30)\end{array}$ & 0.001 & 0.001 \\
\hline $\mathrm{WBC}\left(/ \mathrm{mm}^{3}\right)$ & $\begin{array}{c}5860 \\
(3110-10190)\end{array}$ & $\begin{array}{c}10890 \\
(3560-32480)\end{array}$ & $\begin{array}{c}8450 \\
(3460-22340)\end{array}$ & 0.001 & 0.001 \\
\hline Neutrophils $\left(/ \mathrm{mm}^{3}\right)$ & $\begin{array}{c}3530 \\
(1738-6046)\end{array}$ & $\begin{array}{c}8673 \\
(1032-29686)\end{array}$ & $\begin{array}{c}5484 \\
(1518-21155)\end{array}$ & 0.001 & 0.001 \\
\hline PSI score & & $79.03 \pm 38.91^{\mathrm{e}}$ & & & \\
\hline CURB-65 score ${ }^{\mathrm{d}}$ & & $0.88 \pm 0.85^{\mathrm{e}}$ & & & \\
\hline APACHE II score & & $9.22 \pm 5.57^{\mathrm{e}}$ & & & \\
\hline
\end{tabular}

${ }^{a} \mathrm{P}<0.05$ was considered to be a statistically significant difference. ${ }^{\mathrm{b}}$ The statistical difference was analyzed by Mann-Whitney U test. ${ }^{\mathrm{c}} \mathrm{The}$ statistical difference was analyzed by Wilcoxon signed ranks test. ${ }^{\mathrm{d}} \mathrm{A}$ six point score, one point each for confusion, blood urea nitrogen $>19 \mathrm{mg} / \mathrm{dl}$, respiratory rate $>30 / \mathrm{min}$, low systolic $(<90 \mathrm{mmHg})$ or diastolic $(<60 \mathrm{mmHg})$ blood pressure, and aged $>65$ years. ${ }^{e} \mathrm{Mean} \pm$ standard deviation. CRP, C-reactive protein; WBC, white blood cell; C, controls; UT, patients with CAP prior to treatment; T, patients with CAP following treatment; PSI, pneumonia severity index; APACHE II, acute physiology and chronic health evaluation II.

may be useful markers for inflammation severity. However, the correlation between sAxl and pneumonia has not been examined. Therefore, the plasma sAxl concentrations of CAP patients prior to and following treatment with antibiotics and the normal controls were compared. In addition, it was determined whether the severity correlated with the expression of sAxl or was associated with CAP susceptibility.

\section{Materials and methods}

Participants and diagnosis. This study was performed on a group of 121 individuals, comprised of 61 CAP patients and 60 healthy controls, between February, 2009 and December, 2009. Venous blood samples from CAP patients were obtained through routine venipuncture at Chung Shan Medical University Hospital (Taichung, Taiwan), prior to and following treatment with antibiotics. This study was approved by the Chung Shan Medical University Hospital Institutional Review Board and each patient provided informed consent. The demographic characteristics, comorbidities, symptoms and signs of pneumonia, laboratory results and previous antibiotic treatment of each patient were recorded once they had been admitted. The diagnostic criteria for CAP were based on the guidelines of the Infectious Diseases Society of America/American Thoracic Society (9). The guidelines for CAP diagnosis included a typical infiltration change on chest X-ray films within one day of symptom occurrence and at least one clinical manifestation, including a cough, thick yellow sputum, or a high fever $\left(>37.8^{\circ} \mathrm{C}\right)$; or at least two minor criteria, including tachypnea, dyspnea, pleural pain, chest pain, confusion or disorientation, lung consolidation, or white blood cell (WBC) counts of $>12000$ cells/ml. Exclusion criteria included outpatient status; transfer from another hospital or hospital admission within the previous three weeks; other acute conditions, including pulmonary edema, pulmonary embolism, or malignancy appearing during follow-up; pneumonia caused by tuberculosis or malignancy; severe immunocompromisation, including severe neutropenia (WBC count $<1.0 \times 10^{9}$ cells/1); organ or bone marrow transplant and human immunodeficiency virus infection. Pneumonia severity was assessed using the pneumonia severity index (PSI) (10), acute physiology and chronic health evaluation II (APACHE II) (11) and CURB-65 (12) tests.

Participants and blood specimen collection. In total, 61 CAP patients and 60 healthy individuals, to serve as a control group, were recruited. All CAP patients were treated with empirical antimicrobial agents. Blood samples were obtained from all CAP patients in order to test the WBC, neutrophil cell count, C-reactive protein (CRP) concentration, and plasma concentration of sAxl prior to and following treatment with antibiotics. Blood samples for the control group were also obtained and analyzed. The blood samples were placed in tubes containing EDTA and were immediately centrifuged (3,000 x g, $10 \mathrm{~min})$ and stored at $-80^{\circ} \mathrm{C}$. Depending on the severity of their conditions, CAP patients were treated with effective and common antibiotics, including cefuroxime, ceftizoxime, and clarithromycin. Table I provides a summary of the clinical data and demographics of the patients and controls.

Measurement of WBCs, neutrophils and CRP concentrations. Staff members who were unaware of the source of the samples (blinded to the study) measured WBCs, neutrophils and CRP concentrations by enzyme immunoassays in a clinical laboratory. 
Measurement of plasma sAxl level by enzyme-linked immunosorbent assay (ELISA). ELISA was used to measure the plasma concentrations of sAxl in all blood samples (Aviscera Bioscience, Santa Clara, CA, USA). For each plasma sample, $100 \mu 1$ was directly transferred to the microtest strip wells of the ELISA plate and subsequently incubated for $2 \mathrm{~h}$ at room temperature. Following four washing steps to remove unbound protein, the detection antibody (anti-sAxl; Aviscera Bioscience, Santa Clara, CA, USA) was added and the reaction system was incubated for $2 \mathrm{~h}$ at room temperature. Antibody binding (anti-sAxl) was detected with streptavidin-conjugated horseradish peroxidase and developed with a substrate solution. The reaction was stopped and the optical density was determined using a microplate reader (Molecular Devices, Sunnyvale, CA, USA) set at $450 \mathrm{~nm}$. sAxl concentrations were quantitated with a calibration curve using a human sAxl standard. Each plasma sample was assayed according to the manufacturer's instructions, and the values were within the linear portion of the standard curve.

Statistical analysis. Statistical analyses were performed using SPSS 15.0 statistical software (SPSS Inc., Chicago, IL, USA). All continuous variables were expressed as the mean \pm standard deviation. In order to compare the untreated patients and the healthy controls, the statistical differences between the demographic and clinical data were analyzed using the Mann-Whitney U test for continuous variables not following a parametric distribution. The Wilcoxon signed-rank test was used to compare untreated and treated patients for categorical variables. Linear regression analysis was used to examine the correlation between the $\mathrm{sAxl}$ of all clinical and laboratory variables of CAP patients. $\mathrm{P}<0.05$ was considered to indicate a statistically significant difference in the two-tailed test.

\section{Results}

Demographic and clinical characteristics. Table I provides a summary of the demographic and clinical characteristics. The analysis was based on a sample of 121 individuals; the age and percentage of male participants did not differ significantly between the CAP patients and the control group. The blood cell counts of WBCs and neutrophils and CRP levels were significantly elevated in patients with CAP prior to treatment, compared with the healthy controls $(\mathrm{P}<0.001$; Table I $)$ and patients with CAP following treatment $(\mathrm{P}<0.001$; Table I).

ELISA analysis. Fig. 1 demonstrates the plasma-soluble Axl concentration levels $(\mathrm{pg} / \mathrm{ml})$ of 61 CAP patients and 60 control participants obtained using ELISA analysis. The plasma-soluble Axl concentration level was significantly elevated in CAP patients prior to treatment $(18,143.2 \pm 7,551.5 \mathrm{pg} / \mathrm{ml})$ compared with the controls $(8,328 \pm 1,909.4 \mathrm{pg} / \mathrm{ml})(\mathrm{P}<0.001)$, and decreased significantly following treatment $(14,799.9 \pm 6,762.8 \mathrm{pg} / \mathrm{ml})(\mathrm{P}<0.001)$.

Correlation between plasma-soluble Axl expression and $W B C$ and neutrophil counts, and C-reactive protein levels. No significant correlations were observed with plasma-soluble Axl concentrations (Fig.2). Fig. 3 reveals correlations among PSI, CURB-65, APACHE II scores, and plasma-soluble Axl

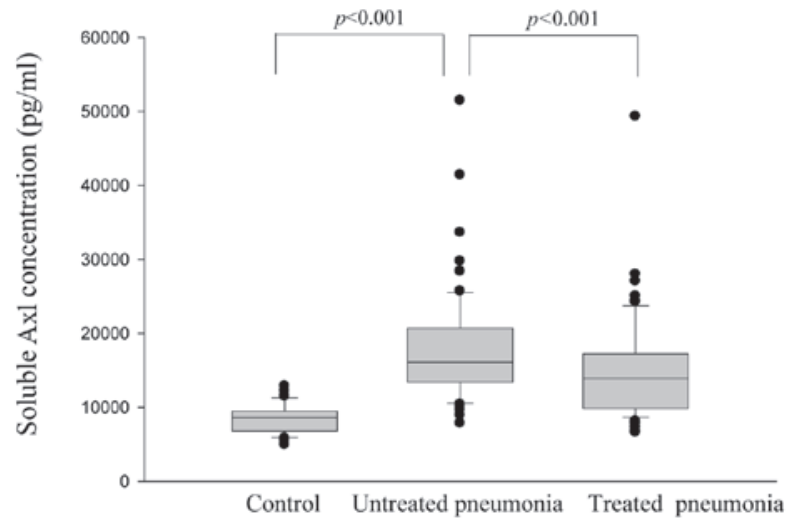

Figure 1. Levels of plasma-soluble Axl concentrations (pg/ml) in 61 patients with community-acquired pneumonia (CAP) and 60 control participants obtained using enzyme-linked immunosorbent assay analysis. The plasma soluble Axl concentration levels were significantly elevated in patients with CAP prior to treatment, compared with participants in the control group $(\mathrm{P}<0.001)$, and decreased significantly following treatment $(\mathrm{P}<0.001)$.

A

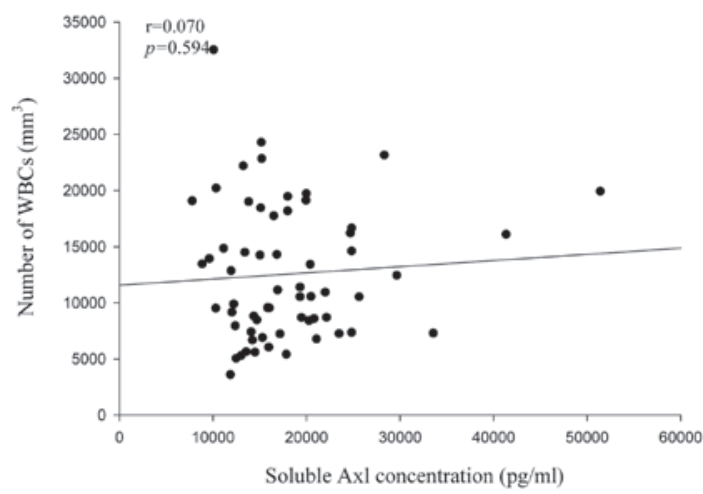

B
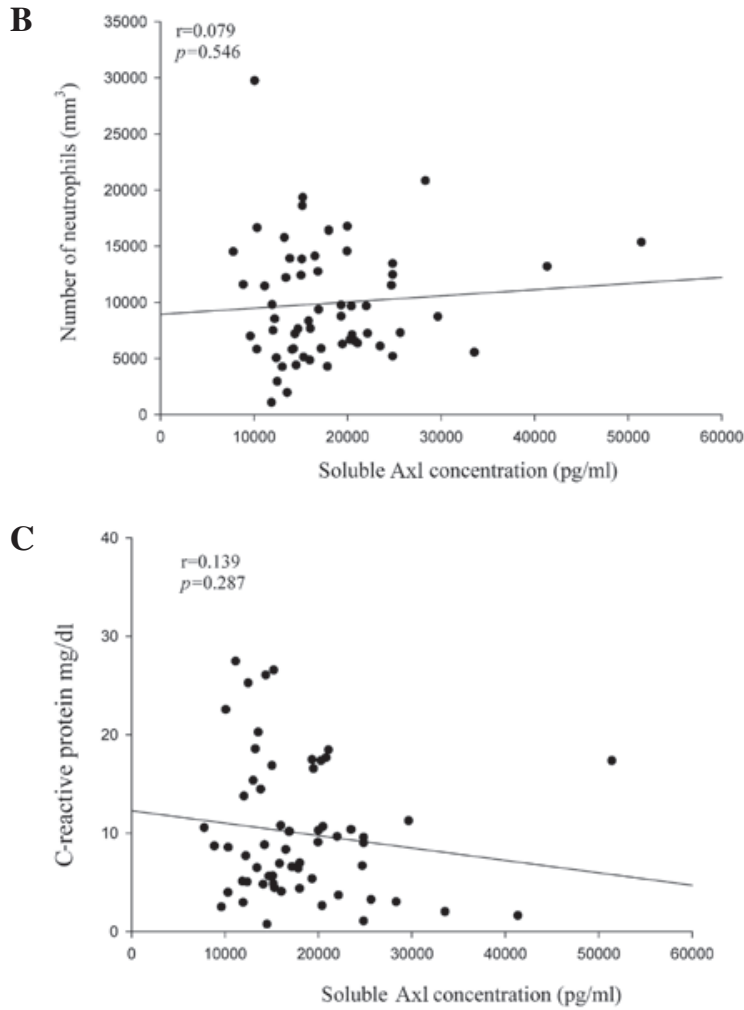

Figure 2. Analysis of the correlation between plasma-soluble Axl expression with white blood cells, neutrophil counts, and C-reactive protein. No significant correlations were observed with plasma-soluble Axl concentrations. 
A

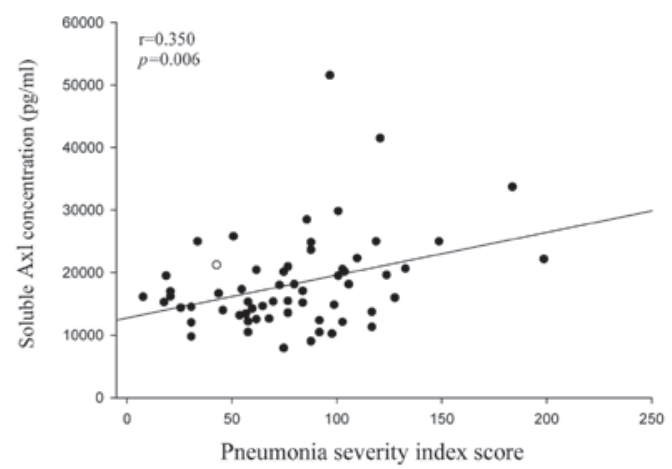

B

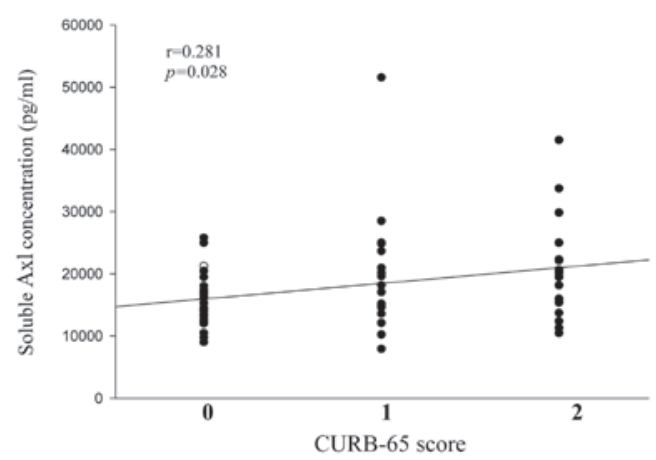

C

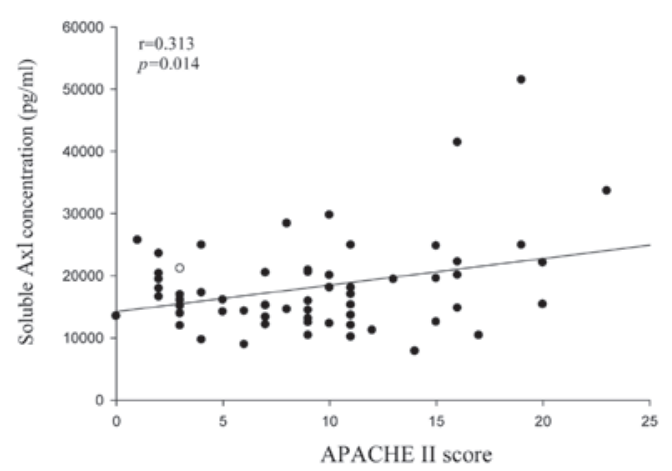

Figure 3. Correlations were observed among the pneumonia severity index (PSI), CURB-65, acute physiology and chronic health evaluation II (APACHE II) scores and plasma-soluble Axl concentrations in community-acquired pneumonia (CAP) patients prior to treatment. Significant correlations were observed between plasma-soluble Axl concentrations and (A) PSI; Spearman correlation coefficients: $r=0.350, P=0.006, n=61$, (B) CURB-65; Spearman correlation coefficients: $\mathrm{r}=0.640, \mathrm{P}=0.028, \mathrm{n}=61$, and (C) APACHE II; Spearman correlation coefficients: $r=0.313, \mathrm{P}=0.014$, $\mathrm{n}=61$.

concentrations in CAP patients prior to treatment. Significant correlations were observed between plasma-soluble Axl concentrations and PSI (Spearman correlation coefficients: $\mathrm{r}=0.350, \mathrm{P}=0.006, \mathrm{n}=61$; Fig. 3A), CURB-65 (Spearman correlation coefficients: $r=0.640, P=0.028, n=61$; Fig. $3 B$ ), and APACHE II (Spearman correlation coefficients: $r=0.313$, $\mathrm{P}=0.014$, n=61; Fig. 3C).

\section{Discussion}

Axl (also known as UFO or Ark) is a $140-\mathrm{kD}$ protein, originally identified in human patients with chronic myelogenous leukemia (13). Axl is a member of the TAM family of receptor
RTKs, consisting of Tyro3 (Sky), Axl, and Mer (13-16). Axl is stimulated by Gas6 (product of the growth arrest-specific gene 6) that was originally observed as a protein expressed by growth-arrested fibroblasts $(7,17)$. The soluble form of Axl (sAxl) may be found in circulation. The Axl receptor is expressed in a wide range of tissues and cell lines (18). Overexpression of the Axl receptor occurs in several human cancer types (16) and is crucial for the growth of human gliomas (19) and gastric cancer (20). Furthermore, several studies have focused on the correlation between sAxl concentrations and inflammation. Ekman et al indicated that sAxl correlates with a number of inflammatory diseases, including SLE and CLI (5,6). In addition, Ekman et al (6) indicated that the sAxl ligands and sAxl concentrations correlate with numerous inflammatory markers in systemic inflammation response syndromes and sepsis. Therefore, the number of studies on the correlation between sAxl and inflammatory diseases (except pneumonia) have increased.

In CAP patients, the correlation among sAxl concentrations, number of WBCs, neutrophils, and CRP levels with regard to severity remains unclear. Therefore, this study examined the changes in the plasma concentration of sAxl, the number of WBCs and neutrophils, and CRP levels in CAP patients. The results revealed that the number of WBCs and neutrophils, and the levels of CRP and sAxl decreased considerably following treatment with antibiotics. However, the number of WBCs, neutrophils and levels of CRP did not correlate with the sAxl levels in CAP patients prior to treatment with antibiotics. This result is similar to that obtained by Lee et al (21), which demonstrated that CRP was not associated with the ligands of sAxl in patients with chronic renal failure. However, Ekman et al (22) indicated that the concentrations of Gas6 and sAxl correlated with CRP in SLE patients with varying degrees of disease activity. Therefore, the correlation between the levels of sAxl and CRP may be controversial in various disease types and various diseases have differing correlations of sAxl and CRP levels. A greater number of samples and experiments are required in order to verify this concept in future studies.

The correlations between plasma sAxl levels and PSI, CURB-65, APACHE II scores in CAP patients remain unclear. These scoring systems are crucial in order to predict the mortality rate of CAP patients as it represents the severity of the disease. Therefore, the plasma sAxl concentrations of CAP patients were further examined prior to treatment with antibiotics in order to determine whether CAP severities correlated with the PSI, CURB-65 and APACHE II scores. The results demonstrated that significant correlations of plasma sAxl concentrations with PSI, CURB-65 and APACHE II score severity, were observed in CAP patients. However, the number of WBCs and neutrophils, and the levels of CRP did not correlate with the severity of CAP patients. Therefore, altered plasma sAxl levels are a trend in inflammation severity and endothelial dysfunction, and plasma sAxl levels may be used as a diagnostic and prognostic serologic marker for CAP instead of the number of WBCs, neutrophils and levels of CRP. This result is similar to that obtained by Ekman et al (5), in which sAxl concentrations correlated positively with the concentrations of known inflammatory markers and predicted future mortality rates in CLI. To the best of our knowledge, 
this is the first study on the correlation of CAP severity with the expression of sAxl.

In conclusion, this study examined the correlation between the plasma concentrations of sAxl and CAP. The results provided valuable information with regard to the plasma concentrations of sAxl in the assessment of CAP severity and demonstrated that the sAxl concentration correlated significantly with antibiotic treatment.

\section{References}

1. Kumar V, Abbas AK, Fausto N, et al: Pathologic Basis of Disease, 8th ed. Elsevier Saunders, Philadelphia, PA, pp710-711, 2009.

2. Chiang TY, Shyu LY, Tsao TC, et al: Elevated plasma matrix metalloproteinase-9 protein and its gene polymorphism in patients with community-acquired pneumonia. Clin Chem Lab Med 50: 449-454, 2011

3. Lee YT, Chen SC, Shyu LY, et al: Significant elevation of plasma cathepsin $\mathrm{B}$ and cystatin $\mathrm{C}$ in patients with community-acquired pneumonia. Clin Chim Acta 413: 630-635, 2012.

4. Houck PM, Bratzler DW, Nsa W, Ma A and Bartlett JG: Timing of antibiotic administration and outcomes for Medicare patients hospitalized with community-acquired pneumonia. Arch Intern Med 164: 637-644, 2004.

5. Ekman C, Gottsäter A, Lindblad B and Dahlbäck B: Plasma concentrations of Gas6 and soluble Axl correlate with disease and predict mortality in patients with critical limb ischemia. Clin Biochem 43: 873-876, 2010.

6. Ekman C, Linder A, Akesson P and Dahlbäck B: Plasma concentrations of Gas6 (growth arrest specific protein 6) and its soluble tyrosine kinase receptor sAxl in sepsis and systemic inflammatory response syndromes. Crit Care 14: R158, 2010.

7. Hafizi S and Dahlbäck B: Gas6and protein S. Vitamin K-dependent ligands for the Axl receptor tyrosine kinase subfamily. FEBS J 273: 5231-5244, 2006.

8. Gheita TA, Bassyouni IH and Bassyouni RH: Plasma concentrations of growth arrest specific protein 6 and the soluble form of its tyrosine kinase receptor Axl in patients with Systemic lupus erythematosus and Behçets disease. J Clin Immunol 32: 1279-1286, 2012.

9. Mandell LA, Wunderink RG, Anzueto A, et al: Infectious Diseases Society of America/American Thoracic Society consensus guidelines on the management ofcommunity-acquired pneumonia in adults. Clin Infect Dis 44: S27-S72, 2007.
10. Fine MJ, Auble TE, Yealy DM, et al: A prediction rule to identify low-risk patients with community-acquired pneumonia. N Engl J Med 336: 243-250, 1997.

11. Knaus WA, Draper EA, Wagner DP, et al: APACHE II: a severity of disease classification system. Crit Care Med 13: 818-829, 1985.

12. Lim WS, van der Eerden MM, Laing R, et al: Defining community acquired pneumonia severity on presentation to hospital: an international derivation and validation study. Thorax 58: 377-382, 2003.

13. Melaragno MG, Wuthrich DA, Poppa V, et al: Increased expression of Axl tyrosine kinase after vascular injury and regulation by $\mathrm{G}$ protein-coupled receptor agonists in rats. Circ Res 83: 697-704, 1998.

14. Ekman C, Site DF, Gottsäter A, Lindblad B and Dahlbäck B: Plasma concentrations of growth arrest specific protein 6 and the soluble form of its tyrosine kinase receptor Axl as markers of large abdominal aortic aneurysms. Clin Biochem 43: 110-114, 2010.

15. Robinson DR, Wu YM and Lin SF: The protein tyrosine kinase family of the human genome. Oncogene 19: 5548-5557, 2000

16. Linger RM, Keating AK, Earp HS and Graham DK: TAM receptor tyrosine kinases: biologic functions, signaling, and potential therapeutic targeting in human cancer. Adv Cancer Res 100: 35-83, 2008.

17. Manfioletti G, Brancolini C, Avanzi G and Schneider C: The protein encoded by a growth arrest-specific gene (gas6) is a new member of the vitamin $\mathrm{K}$ dependent proteins related to protein $\mathrm{S}$, a negative coregulator in the blood coagulation cascade. Mol Cell Biol 13: 4976-4985, 1993

18. O'Bryan JP, Frye RA, Cogswell PC, et al: Axl, a transforming gene isolated from primary human myeloid leukemia cells, encodes a novel receptor tyrosine kinase. Mol Cell Biol 11: 5016-5031, 1991.

19. Vajkoczy P, Knyazev P, Kunkel A, et al: Dominant-negative inhibition of the Axl receptor tyrosine kinase suppresses brain tumor cell growth and invasion and prolongs survival. Proc Natl Acad Sci USA 103: 5799-5804, 2006.

20. Sawabu T, Seno H, Kawashima T, et al: Growth arrest-specific gene 6 and Axl signaling enhances gastric cancer cell survival via Akt pathway. Mol Carcinog 46: 155-164, 2007.

21. Lee IJ, Hilliard B, Swami A, et al: Growth arrest-specific gene 6 (Gas6) levels are elevated in patients with chronic renal failure. Nephrol Dial Transplant 27: 4166-4172, 2012.

22. Ekman C, Jönsen A, Sturfelt G, Bengtsson AA and Dahlbäck B: Plasma concentrations of Gas6 and sAxl correlate with disease activity in systemic lupus erythematosus. Rheumatology (Oxford) 50: 1064-1069, 2011. 